\title{
SKIN REACTIONS TO PRODUCTS OF THE HAEMOLYTIC STREPTOCOCGUS
}

BY

\author{
H. J. GIBSON, M.B., D.P.H., and W. A. R. THOMSON, M.D. \\ (From the Departments of Bacteriology and Medicine, University of \\ Edinburgh.)
}

In the course of an investigation into the aetiological relationship of the haemolytic streptococcus to acute rheumatism, intradermal reactions to an extract of a strain of that organism were carried out on a series of 586 individuals, comprising rheumatic and control groups. Certain of the results as they relate to rheumatism are recorded elsewhere, ${ }^{3}$, and a review of the relevant literature is to be found in those reports. In many cases the skin reaction of the same patient was tested at intervals, and in every case the Dick reaction was carried out and a throat swab cultured for haemolytic streptocooci.

As many apparently anomalous results were noted, the investigation was pursued in order to throw further light on the phenomena of cutaneous allergy to the haemolytic streptococci, and the relationship of these reactions to the Dick reaction, apart from the question of the aetiology of rheumatic fever. The present report is a record of the findings from that point of view. Work of this kind, involving the production of severe and often painful reactions in human subjects, is difficult to carry out on a scale such as would provide results adequate for statistical analysis. Many experiments which showed promise could not be pursued further owing to lack of suitable cases, or the removal from hospital of the patients on whom tests were being carried out.

\section{Methods and reagents.}

The material used for eliciting the reaction was an extract of ground-up streptococci in 0.5 per cent. carbol-saline. The organisms were grown in broth culture, separated and washed three times in sterile saline and once in distilled water. They were then dried at $45^{\circ} \mathrm{C}$. and pulverized in a ball mill. The resulting powder was suspended in 0.5 per cent. carbol-saline and at once centrifuged, the supernatant fluid being retained as the extract. Growth from 100 c.c. of twenty-four hour broth culture was used to produce 10 c.c. of extract which was diluted 1/100 for purposes of the test. A dose of 0.2 c.c. was injected intradermally. This test dose was selected quite arbitrarily as giving marked reactions in some individuals and not in others. Higher dilutions were used but they did not appear to afford any more definite differentiation between positive and negative reactors. Readings were made in twenty-four and forty-eight hours. The reaction reached its maximum in thirty-six hours. 
The strain used was a haemolytic streptococcus (11CX.) isolated from the throat of a rheumatic patient. It was a typical strain of S. pyogenes in its cultural and biochemical reactions and was not related serologically to any of the scarlatinal types of Griffith. The Dick toxin and Dick control used throughout were the commercial products of Burroughs Wellcome Ltd., supplied diluted for use. In many cases repeated tests were made on the same patient at intervals of two to four weeks.

\section{Results.}

The skin reaction to H.S.E. in relation to age.*-Table 1 summarizes the results of the first test on each of the patients of the series. The incidence of positive reactions to H.S.E. increased up to ten to fourteen years and then remained practically constant. The figures for the Dick reaction suggest that the cases of the present series had more than the usual contact with haemolytic streptococci. Thus, the Dick-positive rates in all age groups were unusually low, never reaching 20 per cent. in any group of sufficient size to justify a percentage estimate.

TABLE 1 .

Age incinence of positive SKin REACtions to haEMOlytic Streptococcus eXtract (H.S.E.) aNd to the Dick REAGENTS.

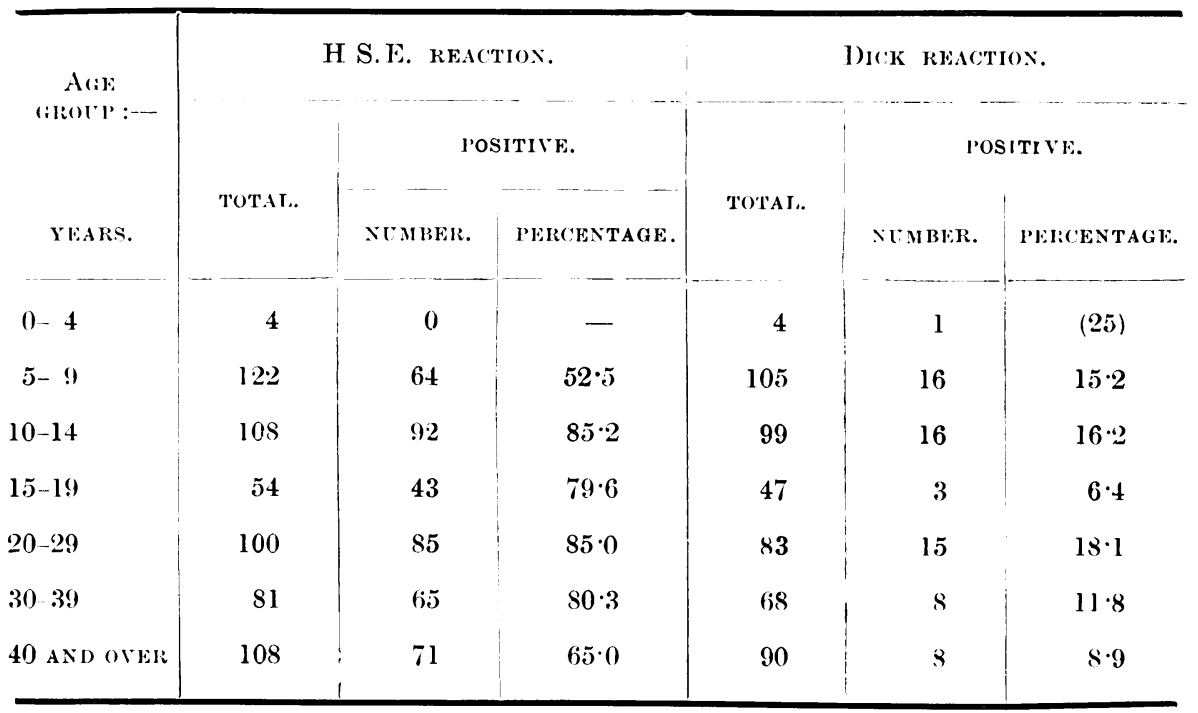

Seasonal incidence of positive H.S.E. reactions. - Tests were carried out throughout four seasons of two consecutive years and in table 2 the results

TABLE 2.

H.S.E. REACTIONS CLASSIFIED ACCORDING TO SEASON.

\begin{tabular}{|c|c|c|c|c|}
\hline $\begin{array}{l}\text { MONTH OF VEAR. } \\
\text { JANIARY, FEPRYARY, MARCH }\end{array}$ & $\ldots$ & $\begin{array}{c}\text { TOTAL } \\
\text { CARRIEI) OIT. } \\
\text { A7\% }\end{array}$ & $\begin{array}{l}\text { TotAL } \\
\text { Positive. }\end{array}$ & $\begin{array}{c}\text { PERCENTALE } \\
\text { POSITIVE. } \\
85 .\end{array}$ \\
\hline Al'RIt, MaY, JUNE & $\ldots$ & 92 & 52 & $5 t i \cdot 5$ \\
\hline Jerx, Autust, Skitembele & $\ldots$ & 111 & 79 & $71 \cdot 2$ \\
\hline Octoble, Notemble, Decembek & $\ldots$ & 352 & 292 & $84 \cdot 1$ \\
\hline
\end{tabular}

*H.S.E. throughout this paper indicates Extract of Haemolytic Streptococcus 11CX, unless otherwise stated, 
are given of skin reactions classified according to the three-monthly period in which they were performed. The majority were carried out during the winter months October to March, but the number (203) performed during the spring and summer was sufficient to show that a significant decline in the incidence of positive reactions occurred, viz. 64.5 per cent., as compared with 84 per cent., during the winter months. These figures refer to repeated tests on the same individuals. When only first tests on each patient are included the comparable figures fall to approximately 60 per cent. and 75 per cent. respectively. These data might suggest that nasopharyngeal and other infections prevalent during winter months influence the general reactivity of a population group to the antigens of streptococcus haemolyticus This suggestion is not supported by the results of throat-swab examinations in the present series (see table 3). It will be noted that the fourth quarter

TABLE 3.

INCIDENCE OF HAEMOLYTIC STREPTOCOCCI IN THROAT SWABS CLASSIFIED ACCORDING TO SFASON.

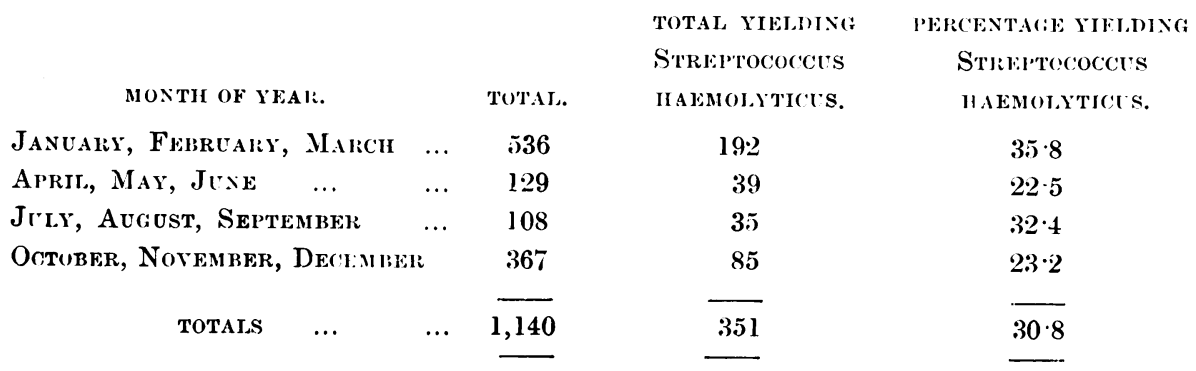

of the year was characterized by a high incidence of positive skin reactions associated with a relatively low incidence of haemolytic streptococci in throat swabs. The number of cases dealt with seemed sufficient to render the figures significant. In this connection it may be noted that the throat swabs were taken at the same time as the skin tests so that the two sets of figures refer to the same individuals throughout.

Correlation of throat culture and skin reaction.-The presence or absence of streptococcus haemolyticus in the throat swab at the time of the test did not appear to influence the skin reaction. In the presence of the organism 72.6 per cent. of 157 cases gave positive skin tests while in its absence the figure was 73.9 per cent. of 402 cases. The streptococci isolated were all examined for haemolysin production in 10 per cent. serum broth and classified as non-haemolytic if it was not demonstrable. The time-lag between throat infection and appearance of allergic skin-reaction may account for the results obtained, past rather than concurrent infection being the important factor. This was shown in scarlet fever by Gibson and McGibbon'. A more likely explanation is that streptococci vegetating on the surface of the pharyngeal mucosa without invading the tissues do not bring about reactivity to their products (see later). 
Reactions to H.S.E. in relation to the Dick test.-Simultaneous Dick tests and tests with H.S.E. were carried out on 492 patients with the results shown in table $4 ; 70 \cdot 1$ per cent. of Dick-positive patients gave also a positive

\section{TABLE 4.}

Correlation of the results of Dick reactions with the H.S.E. Reaction and THE PRESENCE OF STREPTOCOCCUS HAEMOLYTICUS IN THE THROAT SWAB.

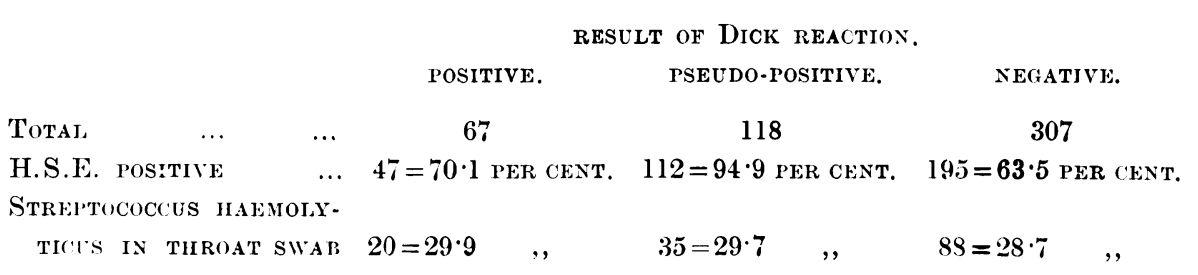

reaction to H.S.E., while the comparable figure among Dick-negative individuals was 63.5 per cent. In view of the small number in the Dickpositive series these figures are not significantly different. This result is somewhat unexpected as it was thought that previous contact with streptococcus haemolyticus leading to the Dick-negative state would also sensitize the patient to the antigens of the organism. Pseudo-positive reactions were obtained in 118 cases (24 per cent.) and nearly all of these (94.9 per cent.) gave a positive H.S.E. reaction, a result which suggests that the constituents of filtrates responsible for the pseudo-reaction are related to the reacting substances in the ground-up bodies of the cocci.

The findings on comparing the results of the Dick test with those of throat-swab cultures (table 4 ) were of considerable interest. There is a remarkable similarity in the incidence of streptococcus haemolyticus in the throats of patients in the three groups, Dick-positive, Dick-pseudo-positive and Dick-negative. Haemolytic streptococci were isolated from 29.9, 29.7 and $28 \cdot 7$ per cent. of these groups respectively. This suggests that the majority of the haemolytic streptococci encountered were either present in the throat as non-invasive commensals or were non-toxigenic.

Repeated tests with H.S.E. in the same patient.-At intervals of two or four weeks repeated skin tests were carried out on 152 patients of our series. The number of tests on each ranged from two to ten. It was at once seen that the skin reactivity of the patients was not constant but varied in an irregular way. In some cases the initial test yielded a marked reaction which tended to show fluctuations at later tests. In others the reaction originally absent, gradually developed on repeated testing. Table 5 illustrates a few of these results taken almost at random from those patients whose stay in hospital was of long duration. The same preparation was used throughout and the technique and reading were uniform over the whole period of the tests. 
No obvious explanation could be found for these variations. The reaction could not be correlated with the clinical condition of the patient. The majority were patients in a convalescent institution and were in fair

\section{TABLE 5.}

Results of repeated skin-testing with H.S.E. Size of raAction (Mean dianeter IN MM.) RECORIDE ALONG WITH PRESENCE OR ABSENCE OF STREPTOCOCCUS HAEMOLYTICCS (H.S.) in throat SWAB (T.S.) AT the time OF tEST.

\begin{tabular}{c|c|c|c|c|c|c}
\hline \multirow{2}{*}{$\begin{array}{c}\text { NUMBER } \\
\text { OF TEST. }\end{array}$} & \multicolumn{2}{|c|}{ CASE 124. } & \multicolumn{2}{c|}{ CASE 126.} & \multicolumn{2}{c}{ CASE 118.} \\
\cline { 3 - 7 } & H.S. E. & H.S. IN & H.S.E. & H.S. IN & H.S.E. & H.S. IN \\
REACTION. & T.S. & REACTION. & T.S. & REACTION. & T.S. \\
\hline 1 & 45 & - & 34 & - & 52 & - \\
2 & 22 & + & 20 & - & 20 & - \\
3 & 41 & - & 53 & - & 33 & - \\
4 & 54 & - & 55 & - & 21 & + \\
5 & 31 & - & 54 & - & 44 & - \\
6 & 30 & - & 31 & - & 20 & - \\
7 & 56 & - & 42 & - & 20 & - \\
8 & 44 & - & 42 & - & 20 & - \\
9 & 31 & - & 0 & - & & \\
10 & 20 & + & 21 & + & & \\
\hline
\end{tabular}

health: the diminution of the reaction could not be attributed to debility. Throat swabs taken at short intervals frequently revealed the intermittent presence of streptococcus haemolyticus but its appearance could not be correlated with the subsequent reactions. Sometimes the reactions increased in intensity, but more frequently were altered or reduced following the finding of the organism. Two possible explanations may be offered: (1) That the skin reactivity of the patient undergoes spontaneous variation. (2) That the test injections themselves altered the reactivity. If the latter explanation is the true one it becomes necessary to postulate that the intradermal injection of a small quantity of antigen in some cases sensitizes the patient while in others it produces the reverse effect. Owing to the fact that no two individuals react in quite the same way it is impossible to control the effect on reactivity of the minimal doses of antigen used for eliciting the tests themselves. 
A small series of patients was tested with repeated intradermal injections of another antigen, viz. normal horse-serum diluted 1/10 (table 6). It was then found that a reaction to horse serum might be clinically identical with

TABLE 6.

RESUlTS OF REPEATED INTRADERMAL INJECTIONS OF NORMAL HORSE SERUM AT INTERVALS. Dose in each c.ase 0.2 c.c. of a $1: 10$ DIlution of SERUm. Figures indicate mean DIAMETER IN MM.

\begin{tabular}{c|c|c|c|c}
\hline DAY OF TEST. & CASE 233. & CASE 232 & CASE 201. & CASE 173. \\
\hline 1 & 0 & 0 & 0 & 0 \\
3 & 18 & 16 & 0 & 12 \\
5 & 23 & 0 & 0 & 1.5 \\
7 & 7.5 & 24 & - & - \\
9 & 73 & 24 & - & - \\
11 & 0 & 35 & - & - \\
13 & 0 & - & - & - \\
15 & - & - & 21 & 75 \\
17 & - & - & 17 & 53 \\
19 & - & - & 35 & 64 \\
21 & - & - & 37 & 67 \\
23 & - & - & 12 & 0 \\
\hline
\end{tabular}

the so-called allergic reactions to bacterial antigens. Appearing in twelve to twenty-four hours and developing to a maximum in thirty to thirty-six hours the lesion produced showed the swollen dark-red centre and peripheral pale margin as described in the case of reaction to H.S.F. The immediate wheal and erythema type of reaction was not observed in any of the fourteen cases so tested. The reactions reached a mean diameter of $90 \mathrm{~mm}$. in the more marked cases.

This observation was confirmed in a series of experiments on guinea pigs in which daily intradermal injections of undiluted H.S.E. produced reactions which fluctuated in an irregular way.

These findings are of considerable importance in regard to skin reactions in general. For example, in the so-called sensitization of animals with subcutaneous or intradermal foci of infection it is customary as a preliminary to test all and to exclude those giving the reaction. Subsequent positive skin-tests are frequently attributed to the sensitizing procedure. It would appear that the intradermal route may be peculiarly effective in producing hypersensitivity to any antigenic substance.

Repeated Dick tests on the same patient._-In the course of these studies the Dick reaction was of secondary interest and all the available cases were also tested with H.S.E. This must be remembered as a possible cause of 
the fluctuations observed. The number of tests which were carried out on the same patient rendered it necessary to use the Dick control only when the reaction to toxin was positive, i.e. of over $10 \mathrm{~mm}$. mean diameter. The control solution was thus injected the day after the test proper since it seemed unlikely that the patient's reactivity would undergo any change in this short period.

The results shown in table 7 are illustrative of observations made over long periods in twenty-three cases. These all showed a marked variability in their reactions to the Dick reagents. The same patient might show an apparently true Dick-positive, a pseudo-positive or a negative reaction on repeated testing, while the paradoxical appearance of a larger reaction to the control fluid than to the test toxin was not infrequently observed. Commonly the test was negative on the first occasion and became pseudopositive at a later date. Case 109 in table 7 illustrates this type. In other cases, exemplified by case 118, the test, while negative at the outset, later showed a difference between the toxin and control reactions which might normally be taken as indicating a positive result. Case 152 (table 7) is of

TABLE 7.

Results of Repeated Dick Reactions CARried oUt at Fortnightly intervals. Figures indicate Mean DiaMeTERS OF THE SKIN REACTIONS IN MM.

\begin{tabular}{|c|c|c|c|c|c|c|}
\hline \multirow{2}{*}{$\begin{array}{c}\text { Test } \\
\text { XIMliek }\end{array}$} & \multicolumn{2}{|c|}{ Case 109} & \multicolumn{2}{|c|}{ CASE 117} & \multicolumn{2}{|c|}{ CASE 129} \\
\hline & $\begin{array}{l}\text { Dick } \\
\text { ToNis }\end{array}$ & $\begin{array}{c}\text { DICK } \\
\text { CONTROI }\end{array}$ & $\begin{array}{l}\text { DICK } \\
\text { TOXIN }\end{array}$ & $\begin{array}{l}\text { Dick } \\
\text { CONTROL. }\end{array}$ & $\begin{array}{l}\text { DICK } \\
\text { TOXIN }\end{array}$ & $\begin{array}{c}\text { Dick } \\
\text { CoNTROL }\end{array}$ \\
\hline 1 & 0) & - & () & - & $1 \pi$ & 11 \\
\hline 2 & $s$ & - & 29 & 0 & 11 & 6 \\
\hline 3 & 32 & 0 & 11 & 23 & 0 & - \\
\hline 4 & 13 & 14 & 9 & - & 0 & - \\
\hline$\tilde{5}$ & 13 & 16 & 8 & - & 13 & 0 \\
\hline (i & 13 & 15 & 9 & - & 24 & $16 i$ \\
\hline 7 & 14 & 19 & 13 & 0 & 19 & $s$ \\
\hline 8 & 12 & 12 & 27 & 12 & - & - \\
\hline 9 & 15 & 14 & 24 & 11 & - & $\ldots$ \\
\hline \multirow[t]{2}{*}{10} & 27 & 17 & & & & \\
\hline & \multicolumn{2}{|c|}{ CASE 114} & \multicolumn{2}{|c|}{ Case 118} & \multicolumn{2}{|c|}{ C.ISE 152} \\
\hline 1 & & - & () & - & 20 & 0 \\
\hline 2 & 20 & 14 & 14 & 0 & $1 !$ & 19 \\
\hline 3 & 18 & 13 & $\tau$ & - & & - \\
\hline 4 & 24 & 19 & i & 一 & 0 & - \\
\hline j & 11 & 0 & 8 & - & 0 & - \\
\hline ii & 7 & - & - & - & $6 j$ & - \\
\hline 7 & 0 & - & o & - & 12 & 14 \\
\hline$s$ & 17 & 10 & 1.) & 8 & & - \\
\hline$!$ & 20 & 11 & 17 & 0 & $2: 3$ & i \\
\hline
\end{tabular}


interest as showing on consecutive tests a ' positive' and 'pseudo-positive' reaction. In all cases the H.S.E. reaction was noted at the time of the tests and while it showed fluctuations these did not correspond either to the reaction to Dick toxin or to Dick control (see table 8).

TABLE 8.

Repeated Dick aNd H.S.E. SKIN teStS ON FOUR Patients to Illustrate the fact THAT NO PARALLELISM EXISTED IN THE RISE AND FALL OF REACTIVITY TO Dick toXiN, Dick control and extract of haemolytic streptococcus. Figures shown are MEAN DIAMETERS IN MM.

\begin{tabular}{|c|c|c|c|c|c|c|c|c|c|c|c|c|}
\hline \multirow{2}{*}{$\begin{array}{c}\text { Test } \\
\text { NUMBER }\end{array}$} & \multicolumn{3}{|c|}{ CASE 151.} & \multicolumn{3}{|c|}{ CASE 166.} & \multicolumn{3}{|c|}{ CASE 114.} & \multicolumn{3}{|c|}{ Case 80.} \\
\hline & $\begin{array}{l}\text { Dick } \\
\text { TOXIN }\end{array}$ & $\left|\begin{array}{c}\text { DICK } \\
\text { CONTROI }\end{array}\right|$ & H.S.E. & $\begin{array}{l}\text { Dick } \\
\text { roxis }\end{array}$ & $\begin{array}{c}\text { DICK } \\
\text { CONTROL }\end{array}$ & H.S.E. & $\begin{array}{l}\text { Dick } \\
\text { TOXIN }\end{array}$ & $\begin{array}{c}\text { DICK } \\
\text { CONTKOI }\end{array}$ & H.S.E. & $\begin{array}{l}\text { DICK } \\
\text { TOXIN }\end{array}$ & $\begin{array}{c}\text { Dick } \\
\text { CONTROI. }\end{array}$ & H.S.E. \\
\hline 1 & 20 & 15 & $\tilde{5} 3$ & 8 & & 42 & 0 & - & 47 & 0 & - & 0 \\
\hline 4 & 13 & 21 & 80 & 0 & & 58 & 20 & 14 & 26 & 0 & - & 40 \\
\hline 5 & 8 & 0 & 81 & 20 & 11 & $2 \pi$ & 24 & 19 & 48 & 13 & 0 & 32 \\
\hline 7 & 25 & 17 & 57 & & & & 20 & 11 & 19 & 27 & 11 & 27 \\
\hline
\end{tabular}

It is not suggested that a marked difference in size between Dick toxin and Dick control reactions indicated a true Dick-positive state. Rather the results suggested that the repeated intradermal inoculations of bacterial extracts and of the Dick reagents were altering the reactivity of the patient's skin to certain constituents in these latter reagents. As with the extract solution itself this reactivity showed an irregular rise and fall. It is of interest that the 'toxin' and 'control' reactions did not run parallel in these circumstances. They differed in their irregular periodicity and the result was the quite irregular appearance of 'true positive,' ' pseudopositive' and ' negative' Dick reactions in the same individual.

At an early stage of the work this question was studied in a series of twenty-eight cases in which apparently true Dick-positive reactions were associated with a strong reaction to H.S.E. Neutralization experiments were carried out with commercial antitoxin. Mixtures were made up of commercial Dick toxin (diluted for use) of 90 per cent. strength, the antitoxin being present in a 1 in 10 dilution in the mixture. Control tests were made simultaneously with Dick toxin alone and serum alone in the appropriate concentrations. Of the twenty-eight cases no less than twentyone reacted strongly to the serum alone and the tests were thus invalidated. 
In the remaining seven the apparently true Dick-positive reaction was neutralized in five and not neutralized in two, while of the seven positive H.S.E. reactions two were neutralized. At the same time commercial antitoxin was shown to neutralize two out of three pseudo-positive Dick reactions. These results suggested that the Dick readings were inaccurate in persons receiving intradermal injections of extract. This subject will be referred to later when neutralization of apparently true allergic reactions is described.

The conditions of these tests were admittedly artificial but the question arises as to whether infection with the haemolytic streptococcus may not act in a way similar to intradermal inoculations of extract and, by altering the skin reactivity, produce anomalous results with the Dick reagents especially at the later age periods when allergic hypersensitivity is liable to develop.

Further questions arise: (1) Whether or not the 'Dick control' solution (heated toxin) as at present used is an adequate control? It appears possible that the heating to which it has been subjected has altered the antigenic character of those proteins other than toxin whose presence in toxin requires to be controlled. (2) How is the pseudo-reacting substance of 'Dick toxin' related to the extract of the haemolytic streptococcus? Apparently intradermal inoculation of extract will sensitize to a constituent of the filtrate and yet the undulations of this pseudo-reaction were not reflected by those of the H.S.E. reaction itself.

An explanation may be that the extract contains a number of antigens one of which is present in the Dick toxin filtrate but that others obscure its effects in tests made with the extract itself. The whole question of skin reactions to such bacterial preparations is obviously a complex one and no hypothesis involving conceptions of simple and clear-cut exotoxin and endotoxin will explain all the observed facts.

The effect of intramuscular injections of H.S.E. on skin reactivity to Dick toxin, Dick control and H.S.E. extract.-Twelve cases were inoculated intramuscularly with extract in order to demonstrate whether or not desensitization could be achieved. It was, of course, realized that the intravenous route would be more suitable but in presence of severe skin reactions this was considered an unjustifiable risk. Intramuscular injections were given at weekly intervals beginning with the skin-test dose, the quantity being gradually increased till in some cases final doses of $1 \cdot 0$ c.c. of undiluted extract were given. It was significant that no local, focal and general reactions were noted after any of the intramuscular injections even in patients who presented large and even alarming skin reactions.

The results showed that no constant desensitizing effect was produced and that the skin reactions to the various reagents fluctuated in a way 
similar to that observed in the absence of intramuscular injections. Ilustrative examples are quoted in table 9.

TABLE 9.

INFLUENCE of intramuscular inJections of H.S.E. ON SKIN REaCtions to H.S.E., Dick toXin AND 'Dick CONTROL.'

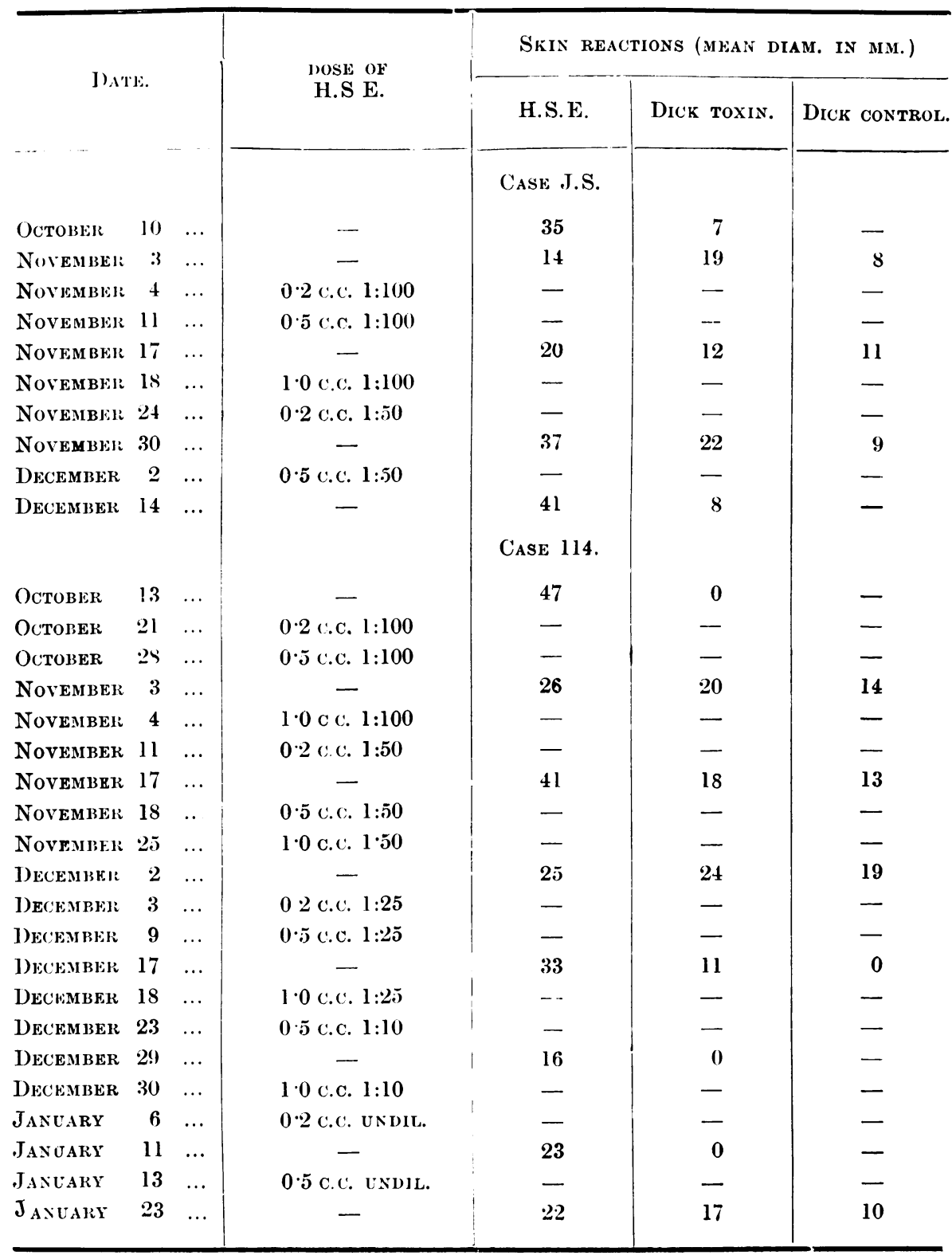

Neutralization of the H.S.E. reaction by antisera.-Scarlatinal antitoxin was the first serum to be employed and in order to make the conditions uniform the Dochez strain of streptococcus was employed for preparing the 
extracts used in these tests. We are indebted to Dr. R. A. O'Brien for his kindness in supplying us with a subculture of the strain used in the preparation of toxin for the manufacture of Burroughs Wellcome commercial antitoxin which was the serum used. Extract-antitoxin mixtures were made up each containing H.S.E. in dilution 1:100, while the antitoxin dilution varied from 1 in 2 to 1 in 100 . With the higher concentration of antitoxin, reactions to serum alone invalidated a number of the tests. In all of eighteen cases where the reaction to extract alone was definite there was no evidence of neutralization by antitoxin.

Further experiments were carried out with an antiserum prepared by immunizing rabbits with H.S.E. derived from the strain 11CX. The animals each received 74 c.c. of undiluted extract over a period of three months. At the end of that time the serum had a low agglutinating titre $(1: 64)$ for the homologous strain and also a low titre precipitating effect. Precipitates were observed up to dilution 1:10 of serum in presence of undiluted antigen and the titre of antigen was approximately the same with undiluted serum. Both serum and antigen were titrated to ascertain if the reaction had an optimal zone at high dilutions but with negative results.

Skin tests were carried out with mixtures containing 11CX extract diluted $1: 100$ along with the serum in dilution $1: 5$, control tests being made with the reagents separately. In seventeen patients tested neutralization was observed in ten, reactions up to $30 \mathrm{~mm}$. in diameter being completely abolished by the serum. Neutralization did not depend on the severity of the reaction to H.S.E. alone as some very weak reactions were not neutralized.

Normal rabbit serum was found to have no neutralizing effect whatsoever on ten patients tested. The possibility of neutralizing a supposed allergic reaction even in only a proportion of test subjects was of such interest that a further experiment was carried out in which the extract of a strain serologically distinct from $11 \mathrm{CX}$ was employed in combination with the anti-11CX-extract serum. The strain used $S_{2}$, was from a case of severe scarlatina complicated by nephritis and was found to be of type 2 (Griffith) while strain 11CX did not belong to any of the Griffith types. Extract of $S_{2}$ in dilution 1:100 was injected with the 11CX extract serum diluted 1:5 into sixteen subjects. All reacted to the extract alone and neutralization failed except in one patient when a weakly positive reaction was neutralized. It appeared that the serum was more efficient in neutralizing reactions to the homologous extract than to others derived from serologically unrelated strains.

Nature of reacting substance in H.S.E.-While it has usually been assumed that the reactions to H.S.E. were due to the presence of nucleo-protein (Coburn ${ }^{1}$, and others) the relatively weak antigenic power of the extract in rabbits suggested that this might not be the case. The active principle was found to be remarkably thermostable. The extract of 11CX, diluted $1: 100$ in 0.5 per cent. carbol-saline was steamed for one hour without loss of potency. Unheated and heated solutions were tested simultaneously on ten patients who reacted and in four the reactions were actually bigger in the case of the heated extract solutions. In one case an increase of more than $2 \mathrm{~cm}$. in the average diameter of the lesions was observed. In the remaining six tests the reaction to extract $\left(100^{\circ} \mathrm{C}\right.$.) was equal to that of the unheated. In the same way a tube of diluted extract (in 0.5 per cent. carbol-saline) was autoclaved at $15 \mathrm{lb}$. pressure for twenty minutes and tests were performed on ten reactors. In six the reaction to autoclaved extract was equal to the test with unheated reagents, while in the remaining four the lesions produced were smaller though the difference in diameter was never more than $1 \mathrm{~cm}$. In no case was the reaction to autoclaved extract 
negative and in six cases the lesion was over $30 \mathrm{~mm}$. in diameter. This degree of thermostability is comparable to that of tuberculin.

Hydrolysis at $100^{\circ} \mathrm{C}$. in alkaline solution $\left({ }_{10}^{\mathrm{N}} \mathrm{NaOH}\right)$ inactivated the reacting property in two hours. The undiluted extract was mixed with an equal volume of $\frac{\mathrm{N}}{5} \mathrm{NaOH}$ and boiled in a flask fitted with a reflux condenser. The extract before and after hydrolysis was tested simultaneously on eight reactors. After one hour the product gave weak reactions in six patients while after two hours all tests were negative.

Precipitation by alcohol.-To 1 c.c. of diluted extract were added 10 c.c. of absolute alcohol and the mixture allowed to stand for two hours in the ice-chest. A flocculent precipitate formed which was centrifuged, washed once in absolute alcohol and resuspended in 10 c.c. of 0.8 per cent. saline in which it was readily soluble. A further dilution of 1:10 was then made for skin tests in parallel with the customary $1: 100$ dilution of untreated extract.

Tests were made on ten patients. In three cases the reaction to the purified preparation was somewhat smaller than the H.S.E. reaction but the difference was slight. In the remainder both lesions were of similar size. Apparently the alcohol-insoluble fraction contained the active principle.

\section{Discussion.}

The results recorded taken as a whole suggest that great caution is necessary in interpreting any intradermal reaction. The possibility of altering the reactivity of the patient by a test inoculation is suggested. The results recorded with horse serum are of interest and they would seem to be applicable to the various diagnostic skin reactions. For example, the repetition of the double intradermal tuberculin test in cattle at short intervals might act as a sensitizing stimulus. The present study suggests that repeated intradermal tests not only sensitize but on a continuation of the series appear to produce desensitization. Thus an irregular wave-like succession of sensitive periods is produced. It is unfortunately impossible to decide whether such variations are inherent in the individual irrespective of the test inoculations or are the result of those.

The result of the Dick tests were somewhat surprising. Only $13.6 \mathrm{per}$ cent. gave positive readings and in the light of subsequent work it appeared likely that not all of these were true toxin reactions. From subsequent studies of fluctuations in the reaction to both the test and control reagents besides the failure to neutralize certain of these reactions by antitoxin, it would appear that some at least were simply the chance association of simultaneous reactivity to some constituent of the 'toxin' fluid with temporary absence of reactivity to a constituent of the 'control' fluid to which the individual might be at other times allergic. These findings have suggested an explanation of those pseudo-reactions when the control test is of greater size than the toxin reaction or even, as in some cases, when the control solution alone gives a reaction. The curves suggest that in the hypersensitive individual the principle responsible for 'pseudo-reactions, to ' toxin' and ' control' may be regarded as different reagents, more or less distinct from one another in so far as skin reactivity is concerned. 
The neutralization of reactions by homologous antiserum was of considerable interest as the effect appeared to be to some extent related to the serological type of the organism. Green ${ }^{5}$ has reported that skin reactions to ' endotoxin' of haemolytic streptococci varied with the serological type of the organism. The skin reaction characteristic of the allergy of infection is not neutralizable and there is no reason to think that any true antigenic toxin was responsible for the lesions produced. Further study of this problem is necessary to determine the mechanism of the neutralization process.

The results throw doubt on the conception of the skin reaction to extracts of haemolytic streptococcus as a true example of the allergy of infection, at least in adults. Myers, Keefer and Oppell ${ }^{6}$ observed the same variations in skin reactivity to a nucleo-protein antigen prepared from extracts of this organism and also failed to discern any correlation between throat-swab cultures and skin reactions.

\section{Summary.}

The results of skin-tests employing the Dick reagents and haemolytic streptococcus extracts, and of simultaneous throat cultures on 586 individuals are described.

No correlation could be discerned between the incidence of the haemolytic streptococcus in the throat and the results of skin reactions to its products.

Repeated tests with 'Dick toxin' and bacterial extract in the same patient revealed a marked variability in the skin reaction to the reagents used. This could not be related to the clinical condition of the patient nor to the presence of the haemolytic streptococcus in the throat. Analogous results were obtained following repeated intradermal inoculation of diluted normal horse serum.

Dick toxin and Dick control reactions did not run parallel in their fluctuations with the result that the patient might present what appeared to be a true Dick-negative reaction, a positive and a pseudo-reaction on successive tests.

Intramuscular injection of extract did not produce local, focal or general reactions and did not appear to influence the skin reaction.

Neutralization of the reaction to bacterial extract was produced in a proportion of cases by an antiserum prepared by immunizing an animal with extract. The reaction to an extract of a serologically unrelated strain was not neutralized by the serum.

Evidence is presented of the marked thermostability of the skin-reacting principle in extracts. Autoclaving a dilute solution at $120^{\circ} \mathrm{C}$. produced little diminution of potency. 


\section{Acknowledgements.}

We are indebted to the honorary staff of the various hospitals and institutions in which the cases were studied, for the full facilities at all times offered to us:-Prof. C. McNeil, Dr. N. Carmichael and Dr. L. H. F. Thatcher of the Royal Hospital for Sick Children, Prof. W. T. Ritchie, Prof. D. Murray Lyon and Dr. J. Eason of the Royal Infirmary, Dr. T. Y. Finlay of the Public Health Department and Lieut.-Col. J. Cunningham and the medical officers of the Astley Ainslie Institution. To Professor T. J. Mackie we are particularly grateful for the advice and guidance received throughout this work.

The investigation has been made possible by personal and expenses grants from the Medical Research Council, and during the latter part of the investigation one of us (W.A.R.T.) has held the Davidson Research Fellowship in Applied Bacteriology.

\section{REFERENGES.}

1. Coburn, A. F., 'The Factor of Infection in the Rheumatic State,' Baltimore, 1931.

2. Gibson, H. J., \& M'Gibbon, J. P., Lancet, Lond., 1932, ii, 729.

3. Gibson, H. J., Thomson, W. A. R., \& Stewart, D., Arch. Dis. Childh., Lond., 1933, VIII, 57.

4. Gibson, H. J., \& Thomson, W. A. R., Edin. Med. J., Edin., 1933, XI, 93.

5. Green, C. A., J. Hyg., Lond., 1935, XXXV, 93.

6. Myers, W. K., \& Oppell, T. W., Jour. Clin. Invest., Lancaster, 1933, XII, 279. 